\title{
Seasonal and age-related variations impacting HPV vaccination uptake in Romania
}

\author{
Oana Streinu-Cercel ${ }^{1,2^{*}}$, Anca Streinu-Cercel ${ }^{1,2}$, Alexandru Rafila $^{2}$, Adrian Pană $^{3}$, Adriana Pistol $^{4}$, Mihai Săndulescu ${ }^{1}$, \\ Adrian Streinu-Cercel ${ }^{1,2}$ \\ From The 9th Edition of the Scientific Days of the National Institute for Infectious Diseases Prof Dr Matei Bals \\ Bucharest, Romania. 23-25 October 2013
}

\section{Background}

We report data regarding the influence of seasonal factors and patient age on the uptake of HPV vaccination in Romania, following the introduction of the vaccine in the national immunization schedule.

\section{Methods}

We prospectively collected data regarding the national immunization schedule between June 2010 and June 2013. For this report, we analyzed the impact of age and seasonal variations on the number of vaccines administered monthly.

\section{Results}

In the studied time span 80,509 HPV vaccine doses were administered in Romania, 46\% $(37,359)$ of these representing the third and final dose of the vaccination regimen. By the end of June 2013, 38,281 women of all ages had completed the full 3-dose HPV vaccination regimen.

The studied time span appears to show a biphasic evolution of the vaccination schedule: between June 2010 and August 2011, absolute numbers were constantly above 1,500 doses per month, while after August 2011 there was an important decrease, with the total number of shots per months constantly below 1,000 but above 150 .

The months with the lowest numbers reported were April $(2,787)$, June $(2,196)$ and July $(1,649) 2011$ in the first time span and September (250), October (245) 2011, September 2012 (186), February (227) and March (205) 2013 for the second time span.

The number of girls undergoing vaccination during June-December 2010 was higher in the lower age groups, with 10,456 girls in the 12-14 years old age group, 13,135
(15-19 years old), 15,723 (20-24 years old), 10,785 (age 24 and over). After 2011, the proportions changed, with the largest numbers of vaccines being administered at increasingly higher ages: 1,671 (12-14 years old), 4,737 (15-19 years old), 8,152 (20-24 years old), 9,800 (age 24 and over) during 2011; 319 (12-14 years old), 598 (15-19 years old), 1,585 (20-24 years old), 1,821 (age 24 and over) during 2012 and 120 (12-14 years old), 215 (15-19 years old), 518 (20-24 years old), 874 (age 24 and over) during JanuaryJune 2013.

\section{Conclusion}

Romania has recorded an interesting shift from the initial school vaccination campaigns to the more recent uptake of vaccine in women of all ages.

\section{Authors' details}

${ }^{1}$ Carol Davila University of Medicine and Pharmacy, Bucharest, Romania. ${ }^{2}$ National Institute for Infectious Diseases "Prof. Dr. Matei Balş", Bucharest, Romania. ${ }^{3}$ Romanian Ministry of Health, Bucharest, Romania. ${ }^{4}$ National Institute of Public Health, Bucharest, Romania.

Published: 16 December 2013

doi:10.1186/1471-2334-13-S1-P28

Cite this article as: Streinu-Cercel et al: Seasonal and age-related variations impacting HPV vaccination uptake in Romania. BMC Infectious Diseases 2013 13(Suppl 1):P28

\footnotetext{
* Correspondence: oanastreinucercel@gmail.com

'Carol Davila University of Medicine and Pharmacy, Bucharest, Romania

Full list of author information is available at the end of the article
} 